\title{
Combination of laser topography measurements with 3D magnetic field mapping for permanent magnets, magnet assemblies and PM ro- tors
}

\author{
Dr. Koen Vervaeke, Magcam NV, Romeinse straat 18, B-3001 Leuven, Belgium
}

\begin{abstract}
As a further development of Magcam's magnetic field camera scanners, we report on a combined stand-alone scanner for very fast measurements of the magnetic field distribution of flat magnets, magnet assemblies and permanent magnet rotors, together with a measurement of the surface topography using a scanned laser triangulation sensor. This allows to correlate features in the measured magnetic field distribution with surface topography features.

The integrated laser distance sensor allows to accurately measure the height, lateral dimensions and full surface map of magnets and magnet assemblies under test. These dimensional measurements complement the high accuracy magnetic field maps measured by the standard integrated 3D magnetic field camera, used for very fast and accurate magnetic field mapping of permanent magnets and magnet assemblies. Both maps can be overlaid, resulting in a correlation between geometric and magnetic data.

Applications of such correlation include magnet height variations as well as lateral positioning errors in magnetic assemblies, surface shape effects, rotor eccentricity in PM rotors etc.

The scanner is controlled by Magcam's powerful MagScope software, which automatically steers the motorized slides, performs the measurements and analyzes the resulting data using powerful algorithms.

The new Combi Scanner is an allround magnetic and dimensional measurement and analysis solution for inspection of permanent magnets, magnet assemblies and PM rotors for use in R\&D labs, QC divisions and production lines and for various applications, such as automotive sensor systems, electric motors, medical devices, consumer electronics and magnet production.
\end{abstract}

\section{Introduction}

Fast and accurate quality inspection of permanent magnets is increasingly important in development, production and quality control of ever more accurate and reliable sensors as widely used in automotive applications, more efficient electric drives for e.g. electric vehicles, new medical devices, consumer electronics and many other magnet applications. Furthermore, in many high-end applications a $100 \%$ magnet quality control is desired, but not feasible with classical magnetic measurement equipment.

In the present paper, first the main components of the Magcam Combi Scanner are described. Subsequently examples of typical measurement results are presented.

\subsection{Magcam 3D Magnetic Field Camera}

Magcam developed the magnetic field camera technology for inspection of permanent magnets, based on a high resolution and high speed quantitative 2D mapping of the magnetic field distribution of a magnet, using a patented sensor chip with an integrated 2D array of microscopic Hall sensors [1-7]. A further development of this technology was a three-axis magnetic field camera ('MiniCube3D', see Figure 1), which makes the three components of the magnetic field distribution available in a $2 \mathrm{D}$ area of $12.7 \mathrm{~mm} \times 12.7 \mathrm{~mm}$, with $0.1 \mathrm{~mm}$ pixel resolution, equaling $128 \times 128=16384$ measurement points [8-10]. A full resolution frame is recorded in less than one second. Small magnets with sizes up to about $10 \mathrm{mmx} 10 \mathrm{~mm}$ can be measured by simply placing the magnet onto the

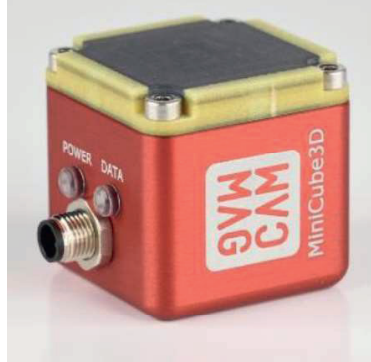

magnetic field camera surface.

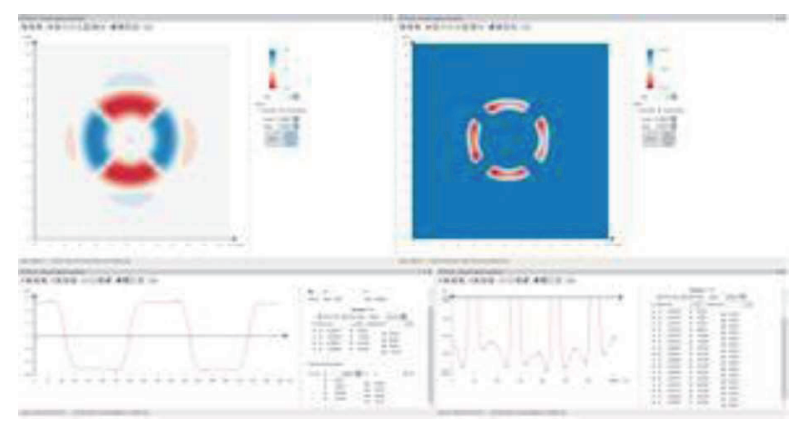


Figure 1: Magcam MiniCube3D magnetic field camera (top) and magnetic field map of a small ring magnet with analysis (bottom)

\subsection{Magcam Combi Scanner}

For larger magnets, magnet assemblies and permanent magnet rotors, Magcam offers its Portal and Rotor Scanners $[1,5-7,10]$, as well as the newest Combi Scanner, combining the Portal and Rotor scanner functionalities into a single Combi Scanner machine.

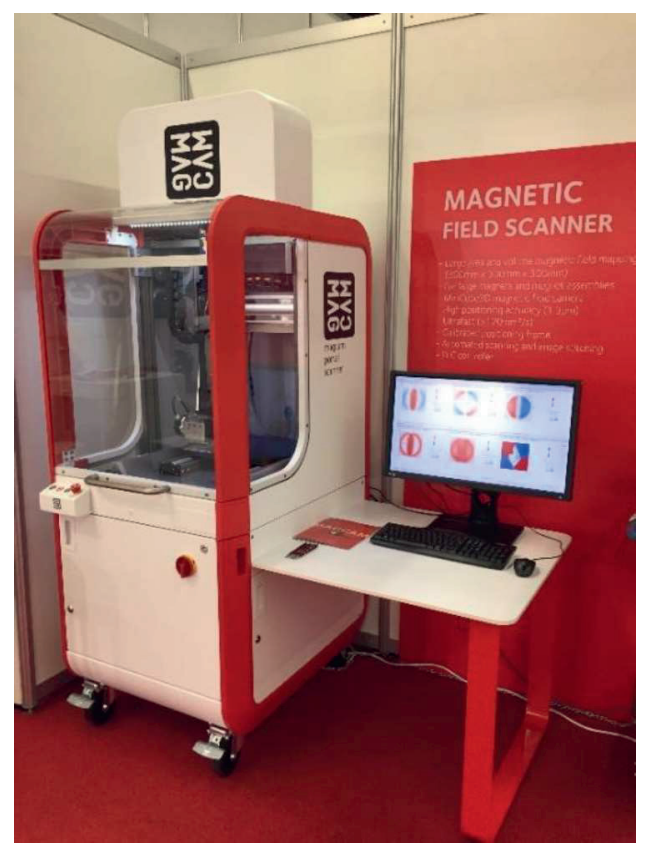

Figure 2: Magcam Combi Scanner

\subsubsection{Portal Scan mode}

To map the 3D magnetic field distribution in a large area and/or a volume, the MiniCube3D magnetic field camera is mounted onto a XYZ scan stage to sequentially measure multiple small-scale magnetic field maps which are subsequently stitched together to obtain a large area image. This configuration is shown in Figure 3.

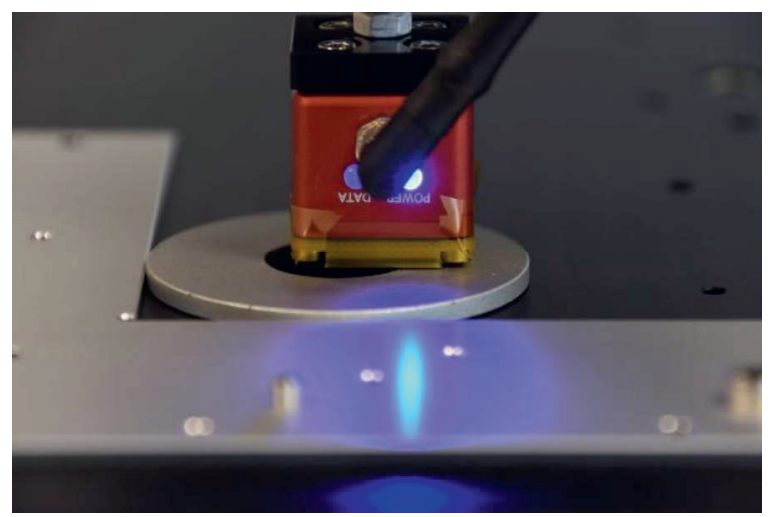

Figure 3: Measuring a flat magnet in Portal Scan mode

\subsubsection{Rotor Scan mode}

For measuring permanent magnet rotors, the MiniCube3D magnetic field camera is rotated over $90^{\circ}$ such that it faces sideways, and a motorized rotary stage is mounted on the sample plate. The rotor under test is mounted vertically in a high accuracy rotary chuck that is mounted on the motorized rotation table, as shown in Figure 4.

Figure 4: Measuring a permanent magnet rotor in Rotor

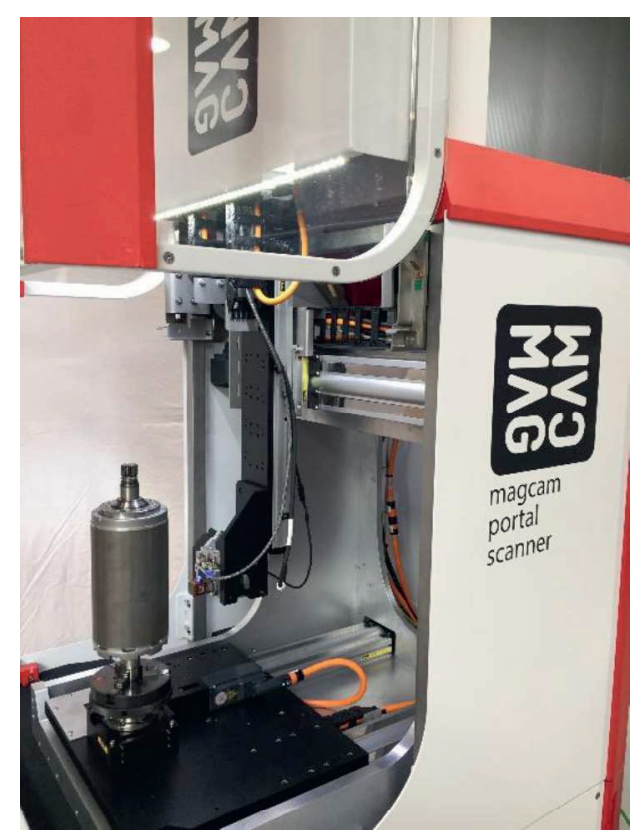

Scan mode

The magnetic field camera is brought in close vicinity to the rotor surface, which is fully automatic thanks to an accurate calibration of the mechanics. During one rotation of the rotor, the magnetic field camera measures a band of $12.7 \mathrm{~mm}$ axial length (this is the length of the MiniCube sensor array). By stitching together multiple bands, the full rotor surface is measured with high axial resolution $(0.1 \mathrm{~mm})$ and arbitrary angular resolution (typically ranging from $0.1^{\circ}$ to $1^{\circ}$ ).

\subsubsection{Laser distance sensor}

A recently developed add-on to the Magcam Portal Scanner and Combi Scanner is a high accuracy Laser Distance Sensor, which allows to measure the height, lateral dimensions and full surface topography map of magnets and magnet assemblies with micrometer accuracy. The laser distance sensor setup is shown in Figure 5. These dimensional measurements complement the high accuracy magnetic field maps measured by the standard integrated $3 \mathrm{D}$ magnetic field camera, used for very fast and accurate magnetic field maps. Both maps can be overlaid, resulting in a correlation between geometric and magnetic data.

The Laser Distance Sensor can be retrofitted on existing PLC-controlled Magcam Portal Scanner setups and takes advantage of the high accuracy mechanics of the scanner, 
converting the scanner in a highly performant hybrid dimensional and magnetic measurement system for in-

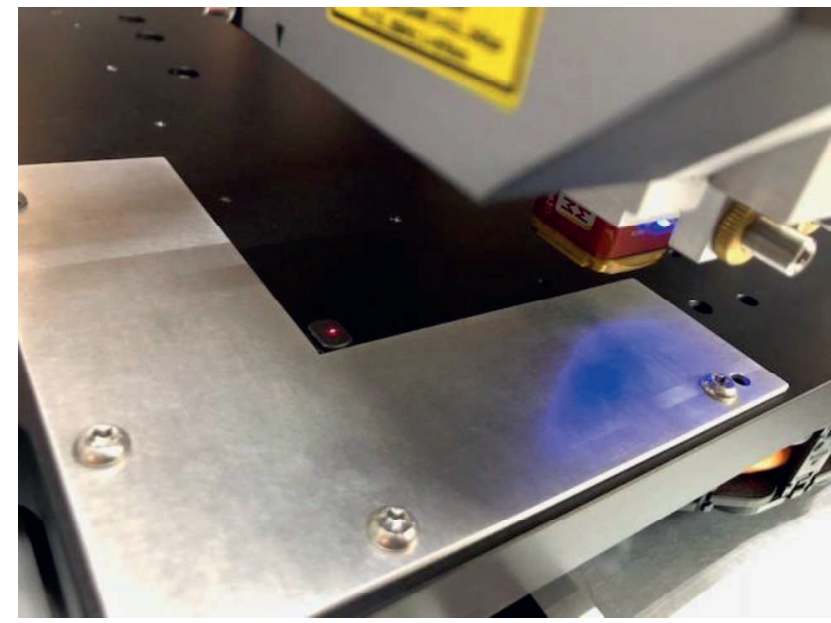

spection of permanent magnets and magnet assemblies. Figure 5: Laser distance sensor for measuring magnet height and sample topography

\section{Measurement results}

\subsection{Flat magnet}

In Portal Scan mode, the Combi Scanner is used to measure flat magnets and magnet assemblies. As an example, the 3D magnetic field distribution of a 2-pole axially magnetized rotary encoder magnet (shown in Figure 5) is measured at a height of $0.5 \mathrm{~mm}$ above the magnet surface. This measurement height is accurately determined by the laser distance sensor first measuring the magnet surface level, combined with accurately calibrated mechanics. The result is shown in , presenting the $\mathrm{Bx}, \mathrm{By}, \mathrm{Bz}$ component distributions in the measurement plane (top color graphs), as well as the full B field, Bxy and azimuthal angle distributions (bottom graphs), which are calculated from the former.

Figure 6, presenting the $\mathrm{Bx}, \mathrm{By}, \mathrm{Bz}$ component distributions in the measurement plane (top color graphs), as well as the full B field, Bxy and azimuthal angle distributions (bottom graphs), which are calculated from the former.

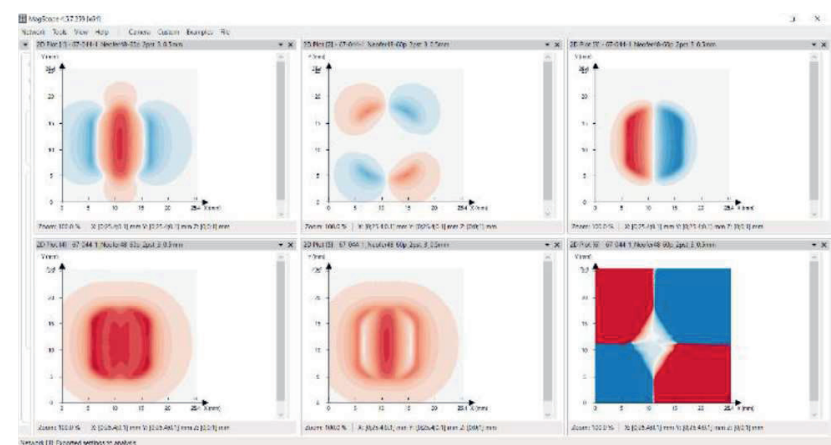

Figure 6: Measured magnetic field distribution of 2-pole rotary encoder magnet. Top: Bx, By, Bz; Bottom: B, Bxy, azimuth angle distributions.

\subsection{Laser topography scan on flat magnet}

The laser distance sensor allows to measure a full topography map of the magnet under test. The result on the

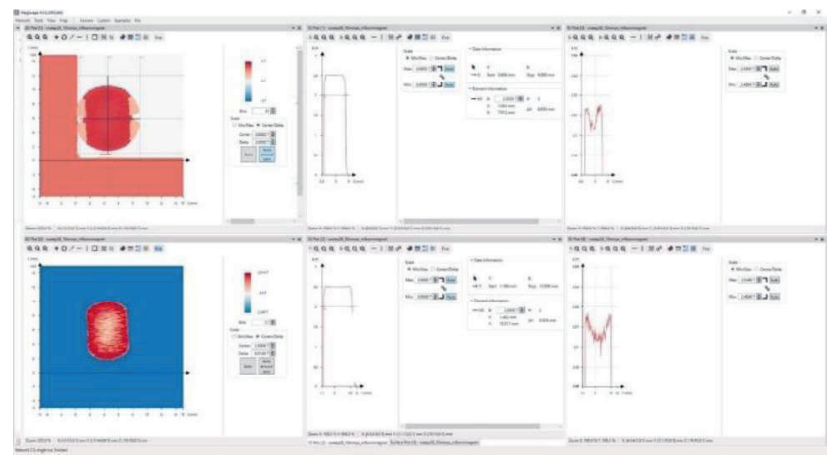

magnet of Figure 5 is shown in Figure 7. The measurement reveals that the magnet is lower in the center and higher towards the edges.

This measured topography can be accurately analyzed using the MagScope software and can be compared to the magnetic field maps of Figure 6.

Figure 7: Magnet surface topography measured by laser sensor scanning

\subsection{Permanent magnet rotor}

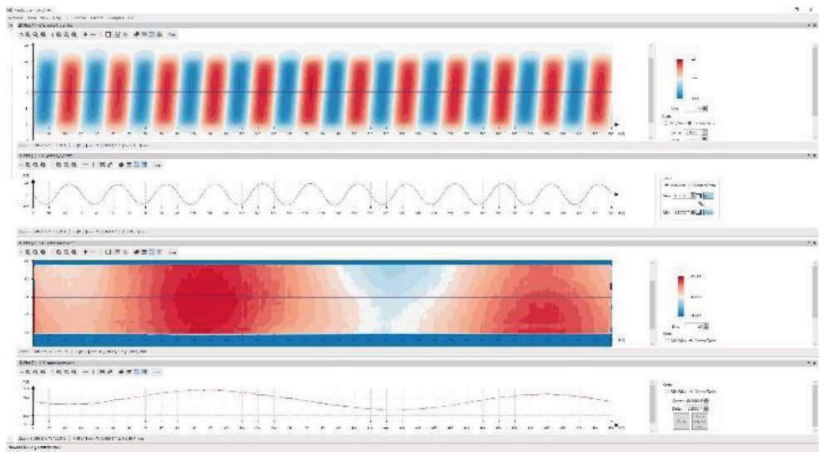

In Rotor Scan mode the 3D magnetic field distribution of permanent magnet rotors is measured as shown in Figure 4, resulting in the magnetic field distribution presented in Figure 8 (top color and line plots, only the radial component is shown).

Figure 8: Radial magnetic field distribution of a permanent magnet rotor (top) and laser topography map of the rotor mantle (bottom)

The magnetic field distribution is shown in cylindrical coordinates and can be analyzed in multiple ways, including automatic finding of zero-crossings and local extremes, pole angle count and analysis and FFT analysis.

\subsection{Laser topography scan on PM rotor}

In Rotor Scan mode the laser distance sensor can be used to measure a full topography map of the rotor under test, as shown in Figure 9. The result of the scan is shown in Figure 8 (bottom color and line plots), showing the measured radius of the rotor as a function of axial and angular positions. The plot shows a second harmonic, indicating a slightly elliptic shape of the rotor. This kind of observations can subsequently be combined with the measured magnetic field distribution in order to analyze 
the source of magnetic features, perform mechanical quality control etc.

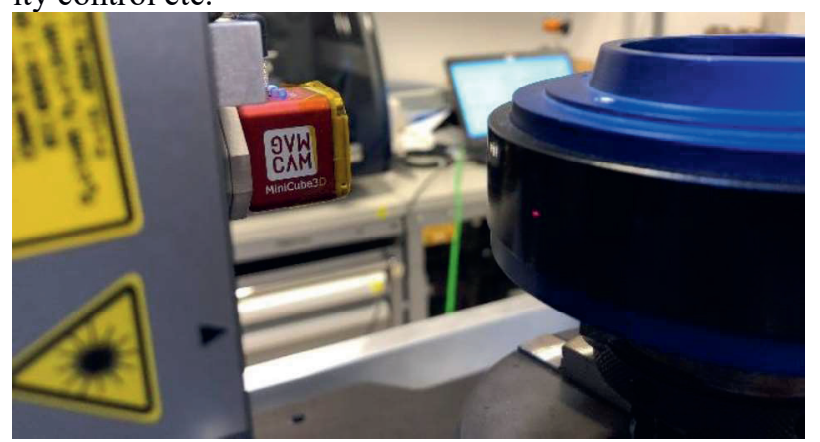

Figure 9: Laser topography measurement configuration of the Combi Scanner in Rotor Mode

\section{Data analysis}

All the measurement data presented in the preceding section can be thoroughly analyzed using Magcam's MagScope measurement and analysis software. Examples of data analysis are available elsewhere [1]. Features include:

- Magnetization vector size and angle deviation analysis

- Interpolation of magnetic field maps for micrometerresolution analysis

- 2D color plots of magnetic field maps

- 2D and 1D (cross-section) region selection in Cartesian and polar coordinates for cut-out and analysis

- Line plots of cross sections in Cartesian or polar coordinates

- Automatic multipole segment detection and measurement of pole sizes/angles

- Statistical analysis on images and cross sections

- Accurate distance/radius/angle measurements on image features (e.g. pole segment sizes)

- Advanced image processing

- Noise reduction and cancellation

- Fourier analysis of cylindrical data (e.g. rotor data)

- Crack detection algorithms

- Integrated Python Scripting Module for e.g. automated pass/fail analysis, integration in production lines and data logging

\section{Conclusion}

We reported on a combined stand-alone scanner for very fast measurements of the magnetic field distribution of both flat magnets or magnet assemblies and permanent magnet rotors. This new 'Combi' scanner is an integration of Magcam's existing Portal and Rotor scanners into a high precision, PLC controlled 4-axis scanner.

We demonstrated the main features of the Combi scanner, including the Portal Scan mode, Rotor Scan mode and the integrated laser distance sensor for accurate dimensional measurements of samples and a design safety housing.

This laser distance sensor allows to accurately measure the height, lateral dimensions and full surface map of magnets and magnet assemblies under test, as well as for permanent magnet rotors, complementing the high accuracy magnetic field maps measured by the standard integrated 3D magnetic field camera.

Both the magnetic field maps and topography maps can be analyzed using the versatile MagScope software, resulting in a thorough and fast analysis of the magnet, assembly or rotor. Many quantitative magnet properties are extracted from the measurement data, making the Magcam system suitable for both R\&D and production of sensor magnets, PM rotors, consumer electronics, medical devices, magnet production and others.

\section{Literature}

[1] www.magcam.com

[2] K. Vervaeke, Conference Proceedings of $15^{\text {th }}$ International Conference on Sensors and Measurement Technology SENSOR 2011, 7.-9.6.2011, Nürnberg, P5.1

[3] K. Vervaeke, Conference Proceedings of 1st International Electric Drives Production Conference (E|DPC 2011), 28-29 September 2011, Nürnberg, ISBN 978-14577-1371-2, pp. 172

[4] K. Vervaeke, Conference Proceedings of 16. GMA/ITG Fachtagung Sensoren Und Messsysteme 2012, 22. und 23. Mai 2012, Nürnberg, 'Magnetic field camera for fast - high resolution inline magnet inspection', 2.3.1

[5] K. Vervaeke, Conference Proceedings of 2nd International Electric Drives Production Conference (E|DPC 2012), 15-18 October 2012, Nürnberg, ISBN 978-14673-3006-0, pp. 186

[6] K. Vervaeke, Conference Proceedings of 16th International Conference on Sensors and Measurement Technology, 14-16 May 2013, Nürnberg (SENSOR 2013), DOI 10.5162/sensor2013/A7.1

[7] K. Vervaeke, Conference Proceedings of 3rd International Electric Drives Production Conference (E|DPC 2013), 29-30 October 2013, Nürnberg, ISBN 978-14799-1102-8, pp. 38

[8] K Vervaeke, Conference Proceedings of 17. ITG / GMA-Fachtagung „Sensoren und Messsysteme", 3.-4. Juni 2014, Nürnberg, P5.2

[9] K. Vervaeke, "3-axis magnetic field camera for ultrafast and high-resolution inspection of permanent magnets" Conference Proceedings of 4th International Electric Drives Production Conference (E|DPC 2014), 30 September - 1 October 2014, Nürnberg, ISBN 978-1-47995010-2, pp. 164

[10] K. Vervaeke, "6D magnetic field distribution measurements of permanent magnets with magnetic field camera scanner', Proceedings of 'SENSOR 2015' Conference, 19-21 May 2015, Nürnberg

[11] K. Vervaeke, "Combined 3D magnetic field camera scanner for both flat permanent magnets and PM rotors", Conference Proceedings of 19. GMA/ITGFachtagung Sensoren und Messsysteme, 26-27 June 2018, Nürnberg 